\title{
Enabling Transmission Through Reentry Plasmas: Simulation of Plasma-SRR Layered Materials
}

\author{
Bruce A. Webb \\ Dept. of Electrical and Computer Engineering \\ University of Arizona, Tucson, Arizona, USA \\ brucewebb@email.arizona.edu \\ Raytheon Missile Systems, Tucson, Arizona, USA \\ bawebb@raytheon.com
}

\author{
Richard W. Ziolkowski \\ Dept. of Electrical and Computer Engineering \\ University of Arizona, Tucson, Arizona, USA \\ ziolkowski@ece.arizona.edu \\ University of Technology Sydney, Ultimo NSW Australia \\ Richard.Ziolkowski@uts.edu.au
}

\begin{abstract}
As a spacecraft enters a planetary atmosphere, severe attenuation of communication signals can occur due to the formation of a plasma sheath around its airframe. The resulting communications blackout has known negative consequences. Early studies have shown that such plasma can be modeled as a negative permittivity material. This feature suggests that a solution may be found in the realm of metamaterials, notably a negative permeability material. In particular, a double negative (DNG) material (i.e., a material with both negative permittivity and permeability) will accommodate travelling waves. We demonstrate that it is possible to introduce a negative permeability metamaterial into the presence of the plasma to form an artificial composite DNG material in order to ameliorate the communication blackout.
\end{abstract}

Keywords-Double negative material, electromagnetic wave propagation, metamaterial, negative permeability, negative permittivity

\section{INTRODUCTION}

When an airframe moves through an atmosphere at hypersonic speeds, it compresses the air in front of it, which generates a great deal of heat. This heat causes ionization of the atmosphere, creating a plasma that effectively coats a majority of the airframe. Depending on the vehicle's size, shape, and speed, it could be completely engulfed in plasma. This plasma sheath can cause severe attenuation of communication and telemetry signals, which is often referred to as a "blackout". The communication blackout can last anywhere from 90 seconds to 15 minutes depending on the trajectory and velocity [1]. More recently, this has also been a problem encountered when spacecraft enter the atmospheres of other planets [2].

Early research suggests that a plasma can be represented by a negative permittivity material [3] for the purposes of studying electromagnetic wave propagation in it. These studies are often facilitated by using the Drude permittivity model to represent the plasma's material properties. The Drude model shows that the real part of its relative permittivity starts out negative at low frequencies, and increases asymptotically to 1 as the frequency increases. The point at which the relative permittivity changes from positive to negative is often defined as the plasma frequency. Negative permittivity materials, often termed epsilon negative (ENG) materials, can only support evanescent waves which decay quickly within them [4].
Likewise, a negative permeability material is generally referred to as a mu negative (MNG) material. It too will only support the presence of evanescent waves.

These single negative (SNG) (i.e., one negative parameter) materials have been engineered using conductive inclusions within a dielectric to produce either a resonant electric or magnetic dipole in the material. The use of split-ring resonators (SRRs) and arrays of conductive rods are examples of inclusions that have been used to produce a MNG or ENG metamaterial, respectively [5].

Furthermore, several types of materials have been engineered to produce both negative permittivity and permeability [6]. These double negative materials (DNG) can support travelling wave propagation. The focus of this paper is exploring DNG materials represented by layers of SNG materials where each layer alternates between ENG and MNG materials. However, the final layer of the multi-layered metastructures must always be an ENG material to represent correctly the outer plasma layer which would be encountered by a high speed vehicle traveling through an atmosphere.

\section{SNG LAYERED METAStructure IN A WAVEgUIDE}

The multilayered metastructure was designed using a waveguide section modeled with the dimensions of a WR187 waveguide for operation in the C-band spectrum. The walls of the waveguide were treated as perfect electric (PEC) sheets. The multiple layers of the SNG materials were located approximately in the middle of the waveguide. The end walls were ports to launch and receive waves propagating along the waveguide. This simulation model is shown in Fig. 1.

The simulations were performed using COMSOL, a fullwave, finite element-based commercial solver. The mesh was composed of tetrahedra. In the free space regions of the waveguide each had a maximum edge length of the wavelength ( $\lambda$ ) divided by 10 . The target frequency was in the neighborhood of $5 \mathrm{GHz}$ at which $\lambda \approx 60.0 \mathrm{~mm}$. The maximum edge length was reduced to $\lambda / 30$ in the SNG layers. An additional 8 layers of continuously varying edge length were introduced to smoothly transition the mesh between the air and SNG regions. 
Initial simulations of the SNG materials were accomplished by specifying the negative permeability or permittivity directly within the material parameters. There were 10 layers, each layer being $1.0 \mathrm{~mm}$ thick. This combination creates an overall

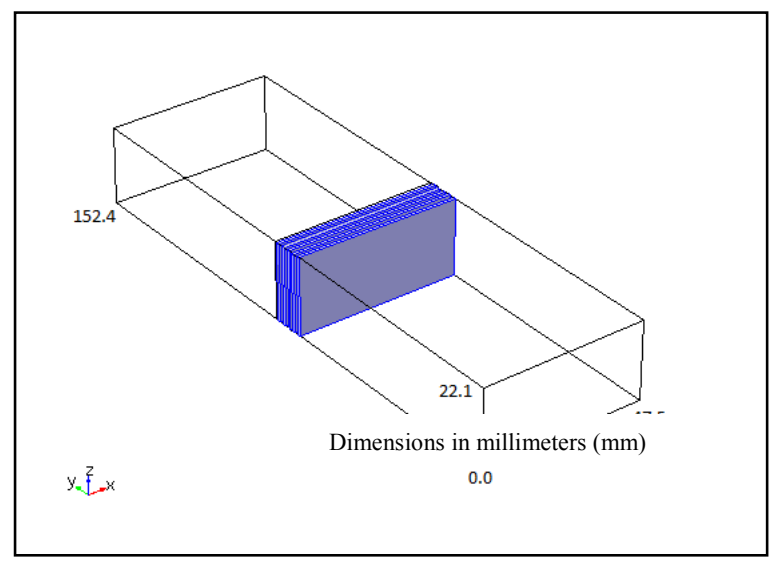

Fig. 1. Wireframe representation of a rectangular waveguide model with embedded SNG layers to simulate normally incident plane waves scattering from a multilayered metastructure.

thickness that is greater than $\lambda / 10$ while the thickness of each layer is less than $\lambda / 10$ to represent the maximum size of a proper metamaterial unit cell. This initial simulation series facilitated the validation of the concept that a metastructure constructed simply by juxtaposing properly selected ENG and MNG layers will support the propagation of electromagnetic waves through it. Moreover, we were able to develop a wideband metastructure that is well matched to air. The associated simulation results are shown in Fig. 2. The scattering $(\mathrm{S})$ parameter $\left|\mathrm{S}_{21}\right|$ is clearly near $0 \mathrm{~dB}$ and $\left|\mathrm{S}_{11}\right|$ is less than $-10 \mathrm{~dB}$ across the entire frequency band of interest.

More physically realizable structures were then considered. The number of layers was reduced. A single $1.0 \mathrm{~mm}$ bulk ENG layer represented the plasma. It was combined with a single $1.0 \mathrm{~mm}$ MNG layer built as a slab of SRR unit cells. The SRR unit cell was designed to be resonant within the $\mathrm{C}$-band region. The dimensions of the SRR design are shown in Fig. 3 and listed in Table 1. The boundaries of the waveguide dimensions were used to limit the SRR material dimensions as shown in Fig. 4. The planar orientation of the SRRs shown in Fig. 3 facilitated their resonant excitation by the electric field of the wave exciting them. It also represents the realization that one would like to have as thin a MNG layer as possible in practice and to enable further studies of a "thin" multilayered configuration.

\section{SIMULATION RESULTS}

According to [7], matching the layers to air requires attention to the material thicknesses, to the ratio of the negative permittivity to negative permeability values, and to the angle of incidence onto the SNG material. Initial results, shown in Fig. 5 , indicate that a much narrower pass-band occurs. This feature is due to both the resonant behavior of the SRRs and to their dispersive properties. When the negative permeability of the SRR material is equal to the negative permittivity of the ENG material, there is a minimum in the $\left|S_{11}\right|$ values and a corresponding maximum of the $\left|\mathrm{S}_{21}\right|$ values.

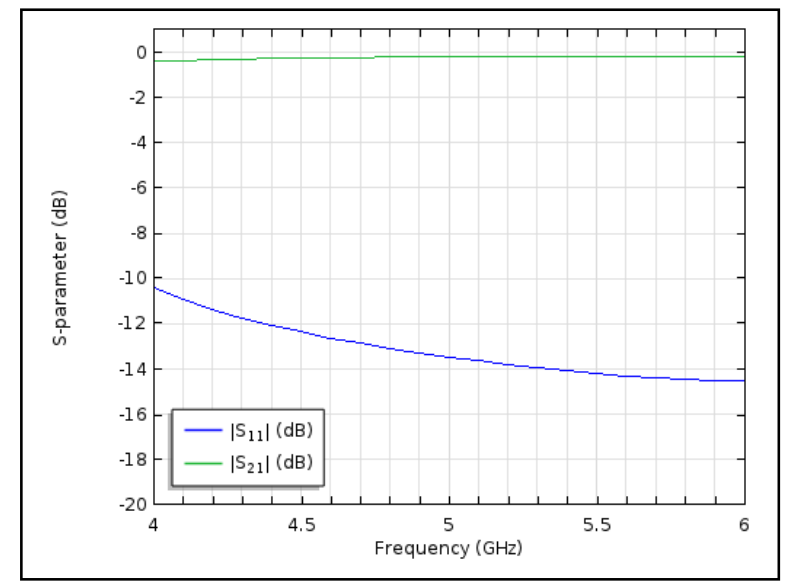

Fig. 2. Simulated S- parameters of the multilayered-SNG metastructure.

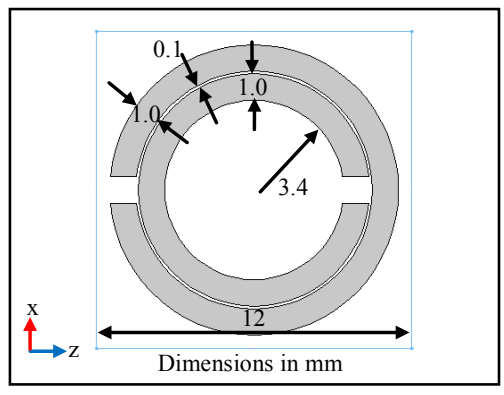

Fig. 3. SRR unit cell.

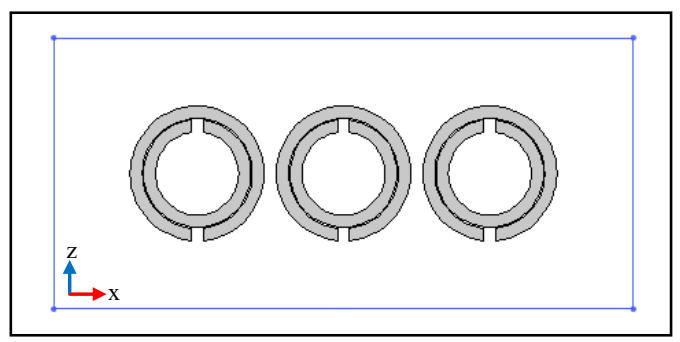

Fig. 4. SRR material within waveguide boundaries.

There is also a very weak resonance at a lower frequency due to the bianisotropic nature of the SRRs [8]. The magnetic field would need to be oriented orthogonal to the plane containing the SRR elements for a strong resonance at this lower frequency. However, this configuration is undesirable since, as noted, a thin metastructure is desired.

Because the plasma frequency depends on the plasma density, it is desirable to study the effects of changing the plasma parameters. The negative permittivity of the ENG material was adjusted from -1.5 to -3 in steps of 0.5 . Fig. 6 shows the results for three cases. As the permittivity is adjusted, the negative parameters become equal at different frequencies, thus impacting the S-parameter values.

To investigate the effects of a change in material thickness, the ENG material was maintained to have a constant $1.0 \mathrm{~mm}$ thickness while the SRR material thickness was varied from 
1.0 to $2.0 \mathrm{~mm}$ in $0.2 \mathrm{~mm}$ steps. This resulted in a small shift in the resonant frequency and a large change in the $\left|S_{11}\right|$ values.

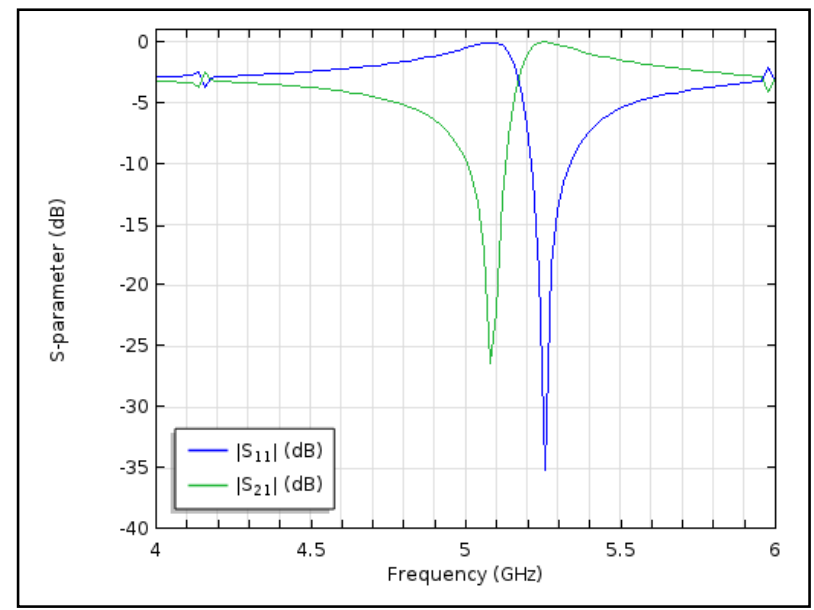

Fig. 5. Simulated S-parameters for the SRR-ENG layered metastructure.

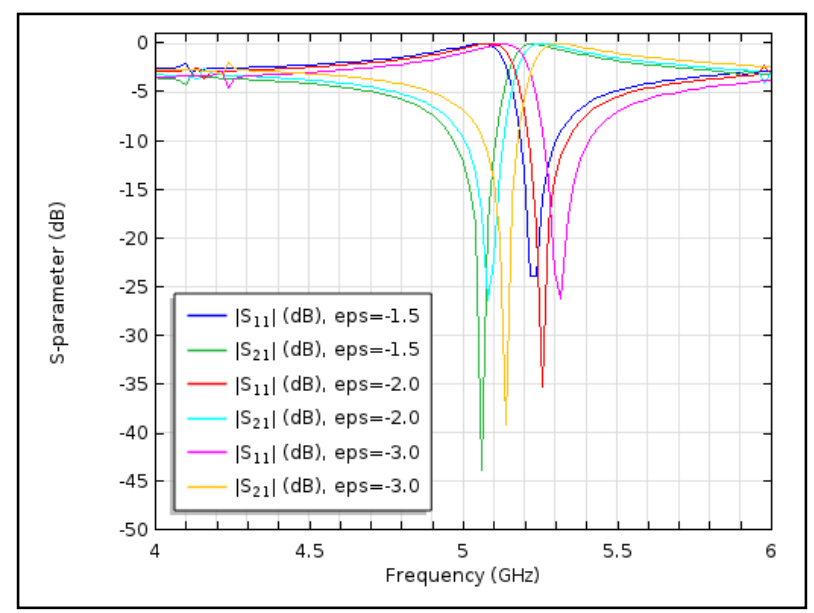

Fig. 6. Simulated responses to variations in the ENG negative permittivity

\section{CONCLUSIONS}

When considering the communication blackout issue associated with hypersonic vehicles, it is useful to think of the plasma sheath as a negative permittivity material. With known metamaterial features [9], solutions to the blackout phenomenon are possible. Examining a combination of a SRRbased material and the ENG plasma layer demonstrate conclusively that a metastructure can be designed to ensure propagation through the plasma. However, for a solution to be valid over an entire set of vehicle trajectories and hypersonic speeds, variations of the plasma parameters must be taken into account. Changes in the plasma density cause a change in the plasma material permittivity which, in turn, shifts the resonant frequency of a fixed system. Consequently, a shift in the SRR properties will have to follow this trend.

The parameters that can be adjusted to solve this problem are suggested in [7]. As reported, we have currently examined changing the ENG plasma parameters and the thickness of the
SRR-based MNG layer. While there was a $10-\mathrm{dB}$ change in the minimum $\left|\mathrm{S}_{11}\right|$ value as the permittivity of the plasma material changed from -3.0 to -1.5 , there was a $15-\mathrm{dB}$ change in the minimum $\left|\mathrm{S}_{11}\right|$ as the SRR dielectric thickness was altered from 1.0 to $2.0 \mathrm{~mm}$. This suggests the possibility that the change in permittivity could be compensated within the range of potential permeability values.

To attain a dynamically tunable system to meet the situations in which the plasma density would be changing, several options are available. The permeability of an SRR can be tuned in real time by adding an adjustable capacitance (i.e., a varactor) to it as discussed in [10]. Furthermore, although a waveguide was used for the initial simulations to simplify them, tunability could also be realized by physically adjusting the distance between the radiating antenna and the MNG material in its near field. Additional explorations of the various antenna-metasurface configurations that would lead to properly excited layered DNG material will help to understand the variables that contribute to the overall system efficiency while attempting to maintain a constant level of transmission through a plasma material with variable permittivity. A variety of simulations of tunable configurations are in currently in progress. The outcomes of these studies will be described in our presentation.

\section{REFERENCES}

[1] R. A. Hartunian, G. E. Stewart, S. D. Fergason, T. J. Curtiss, R.W. Seibold, "Causes and mitigation of radio frequency (RF) blackout during reentry of reusable launch vehicles." Contractor Rep. ATR-2007 (5309)1, Aerosp. Corp., 2007.

[2] D. D. Morabito, "The spacecraft communications blackout problem encountered during passage or entry of planetary atmospheres." IPN Progress Report 42-150, Aug. 2002.

[3] W. Rotman, "Plasma simulation by artificial dielectrics and parallel-plate media", IRE Trans. on Ant. and Prop., vol. 10, no. 1, pp. 82-95, 1962.

[4] C. Balanis, Advanced Engineering Electromagnetics, 2nd ed, Hoboken, NJ: John Wiley \& Sons, 2012, pp.227-245.

[5] R. Marques, F. Martin, M. Sorolla, Metamaterials with Negative Parameters: Theory, De sign, and Microwave Applications Hoboken, NJ. Wiley-Interscience, 2008.

[6] R. W. Ziolkowski, "Design, fabrication, and testing of double negative metamaterials" IEEE Trans. on Ant. and Prop. 51, no. 7, pp. 1516-1529, July 2003.

[7] A. Alu, N. Engheta "Pairing an epsilon-negative slab with a mu-negative slab: Resonance, tunneling and transparency" IEEE Trans. On Ant. And Prop. 51, no. 10, pp. 2558-2571, Oct. 2003.

[8] R. Marques, F. Medina, R. Rafii-El-Idrissi, "Role of bianisotropy in negative permeability and left-handed metamaterials" Phys. Rev. B 65, 144440, pp. 1-6, Apr. 2002.

[9] N. Engheta and R. W. Ziolkowski, Metamaterials: Physics and Engineering Explorations, Hoboken, NJ: IEEE-John Wiley \& Sons, 2006.

[10] I. Gil, et al, "Varactor-loaded split ring resonators for tunable notch filters at microwave frequencies" Electronics Letters. 40, no. 21, Oct. 2004 